Article

\title{
Genome-Wide Identification, Expression Profile of the TIFY Gene Family in Brassica oleracea var. capitata, and Their Divergent Response to Various Pathogen Infections and Phytohormone Treatments
}

\author{
Xing Liu ${ }^{1,+}$, Cunbao Zhao ${ }^{2,+}$, Limei Yang ${ }^{2}$, Yangyong Zhang ${ }^{2}$, Yong Wang ${ }^{2}$, Zhiyuan Fang ${ }^{1,2, *}$ \\ and Honghao $\mathrm{Lv}^{2}$,*(iD) \\ 1 Germplasm Innovation in Northwest China, Ministry of Agriculture, College of Horticulture, \\ Northwest A\&F University, Yangling 712100, Shanxi, China; 13161176649@163.com \\ 2 Key Laboratory of Biology and Genetic Improvement of Horticultural Crops, Ministry of Agriculture, \\ Institute of Vegetables and Flowers, Chinese Academy of Agricultural Sciences, Beijing 100081, China; \\ cunbaozhao@163.com (C.Z.); yanglimei@caas.cn (L.Y.); zhangyangyong@caas.cn (Y.Z.); \\ wangyong03@caas.cn (Y.W.) \\ * Correspondence: fangzhiyuan@caas.cn (Z.F.); lvhonghao@caas.cn (H.L.); Tel.: +86-010-6213-5629 (H.L.) \\ + These authors contributed equally to this work.
}

Received: 31 December 2019; Accepted: 22 January 2020; Published: 24 January 2020

\begin{abstract}
TIFY, a plant-specific gene family with the conserved motif TIF[F/Y]XG, plays important roles in various plant biological processes. Here, a total of 36 TIFY genes were identified in the Brassica oleracea genome and classified into JAZ (22 genes), TIFY (7 genes), ZML (5 genes), and PPD (2 genes) subfamilies based on their conserved motifs, which were distributed unevenly across nine chromosomes with different lengths (339-1077 bp) and exon numbers (1-8). Following phylogenetic analysis with $A$. thaliana and B. rapa TIFY proteins, ten clades were obtained. The expression of these TIFY genes was organ-specific, with thirteen JAZ genes and two PPD genes showing the highest expression in roots and leaves, respectively. More importantly, the JAZs showed divergent responses to various pathogen infections and different phytohormone treatments. Compared with the susceptible line, most JAZs were activated after Plasmodiophora brassicae infection, while there were both induced and inhibited JAZs after Fusarium oxysporum or Xanthomonas campestris infection in the resistance line, indicating their probably distinct roles in disease resistance or susceptibility. Further, the JAZs were all upregulated after MeJA treatment, but were mostly downregulated after SA/ET treatment. In summary, these results contribute to our understanding of the TIFY gene family, revealing that JAZs may play crucial and divergent roles in phytohormone crosstalk and plant defense.
\end{abstract}

Keywords: Brassica oleracea; TIFY; JAZ; phytohormone; disease resistance

\section{Introduction}

The TIFY family is plant-specific and encodes transcription factors (TFs), which was previously annotated as zinc-finger $\left(\mathrm{C}-\mathrm{X}_{2}-\mathrm{C}-\mathrm{X}_{20}-\mathrm{C}-\mathrm{X}_{2}-\mathrm{C}\right)$ protein expressing in the inflorescence meristem (ZIM) family [1]. AT4G24470, which encodes a putative TF that contains the CCT domain and the C2C2-GATA zinc finger domain, was the first gene to be characterized as having a ZIM domain [2]. Then, 29 different Arabidopsis loci encoding proteins containing GATA-like zinc fingers were identified by BLAST searches [3]. However, ZIM and two ZIM-like proteins (ZML proteins), ZML1 (AT3G21175) and ZML2 (AT1G51600), belonged to a different group from the other typical GATA-type proteins, as determined by phylogenetic analysis [3,4]. In addition, the ZIM family was uncharacterized in Pfam or InterPro 
databases, and its definition was confusing [5,6]. In response to these inconsistencies, Vanholme et al. (2007) used 'TIFY' instead of ZIM to indicate the most conserved amino acid pattern TIF[F/Y]XG (where $X$ represents any amino acid) [7].

Based on the different domain architectures, the TIFY family was classified into four subfamilies: TIFY, ZML, PEAPOD (PPD), and Jasmonate ZIM (JAZ) [8]. The TIFY subfamily contains only the TIFY domain, while the ZML subfamily also contains the CCT and ZML domains. The PPD subfamily contains a unique N-terminal PPD and a truncated Jas domain, and the JAZ subfamily has the C-terminal Jas domain (also named the CCT_2 domain in Pfam). To date, TIFY genes have been identified in many species, such as 18 TIFY genes in Arabidopsis [7], 20 in rice [7], 27 in maize [8], 49 in wheat [9], 18 in pigeon pea [10], 24 in Populus trichocarpa [11], and 30 in apple [12]. Moreover, functional research on TIFY family genes has been performed. ZIM (AT4G24470) has been found to regulate petiole and hypocotyl elongation by mediating cell elongation [4], while ZML2 (AT1G51600) acts as a transcriptional repressor in lignin biosynthesis in maize [13]. PPD1 (AT4G14713) and PPD2 (AT4G14720) are involved in the coordination of leaf growth, and their loss-of-function mutations show leaf enlargement and result in dome-shaped leaves, rather than flat leaves [14]. However, owing to the critical role of JAZ genes in the jasmonic acid (JA) pathway, the JAZ subfamily is clearly the best-characterized member of the TIFY family and has received extensive functional research $[15,16]$.

CORONATINE INSENSITIVE 1 (COI1), the F-box component of the $\mathrm{E}_{3}$ ubiquitin ligase complexes $\left(\mathrm{SCF}^{\mathrm{CO} 1}\right)$, is the primary JA receptor $[17,18]$. MYC2, the basic helix-loop helix (bHLH) TF, is a key transcriptional activator of responses to JA [19]. However, the link between COI1 and MYC2 was the major question and unidentified until the discovery of the key functions of AtJAZ1 (AT1G19180) [20] and AtJAZ3 (AT3G17860) in JA-mediated responses [21]. JAZ proteins are direct targets of COI1 with the $C$ terminus Jas domain, and JA-Ile or coronatine could promote these interactions and induce the degradation of JAZ proteins by the $26 \mathrm{~S}$ proteasome in a SCFCOI1-dependent manner [21,22]. MYC2 also interacted with the C-terminal domain of JAZ proteins and was able to induce a set of JA-responsive genes after the degradation of JAZ proteins [21]. MYC3 and MYC4, two other MYC2-related bHLH TFs, have been proven to be able to interact with the majority of JAZ proteins [23]. In addition to MYC transcription factors, MYB TFs (MYB21/MYB24/MYB75/GL1) targeted by JAZ repressors were identified by yeast two-hybrid screens and confirmed in vivo [24,25].

Brassicaceae (alternative name Cruciferae) is a family with numerous economically valuable members, including vegetables, oil crops, medicinal plants, and ornamental plants. Among these species, genome-wide identification of the TIFY gene family has been carried out in Arabidopsis and Brassica rapa $[7,26]$. Cabbage (Brassica oleracea var. capitata L.), an important Brassica vegetable crop of Cruciferae, is cultivated worldwide. In the field, cabbage inevitably suffers from a variety of diseases. Cluboot caused by the protists Plasmodiophora brassicae and Fusarium wilt caused by Fusarium oxysporum f. sp. conglutinans are major soil-borne diseases in cabbage [27,28], and cabbage black rot caused by Xanthomonas campestris pv. campestris is a bacterial disease that has caused increasing damage in recent years [29]. In addition, downy mildew, head rot, and soft rot disease also threaten the cultivation of cabbage to some extent [30-32]. Recent transcriptome studies have shown that plant hormones participate in the regulation of cabbage disease resistance. Ning et al. (2019) found that the salicylic acid (SA) signalling pathway was induced, while the JA signalling pathway was repressed, in the resistant cabbage genotype after P. brassicae infection [27]. Xing et al. (2016) found that the JA and ET signalling pathways and SA-dependent systemic acquired resistance (SAR) play important roles in cabbage resistance to F. oxysporum [33].

In this study, the TIFY family genes in cabbage were identified, and their structure, evolution, chromosome location, and expression characteristics were analysed simultaneously. In view of the key roles of JAZ proteins in JA signalling pathway, the expression patterns of cabbage JAZ genes after exogenous phytohormone treatments and inoculation with different pathogens were analysed further. The results of this study contribute to a better understanding of the TIFY gene family, help to reveal 
the role of JAZ genes in phytohormone crosstalk and plant defense, and provide valuable information for future functional studies.

\section{Materials and Methods}

\subsection{Identification of the B. oleracea TIFY Family Genes}

The genome sequencing of the B. oleracea var. capitata line 02-12 (02-12 genome hereinafter, http://brassicadb.org/brad/) has been completed [34]. However, according to recent studies, there were many assembly errors and incomplete annotations in the 02-12 genome obtained by next-generation sequencing [35-38]. Therefore, the newly released genome of the doubled haploid B. oleracea var. capitata line D134 (D134 genome hereinafter, https://db.cngb.org/search/project/CNP0000469/; manuscript under review) based on the single molecule real-time (SMRT) sequencing methods was used for the identification of $B$. oleracea TIFY family genes.

Based on the annotation information regarding the gene structure, the CDS sequences of all cabbage genes were extracted from the genome and translated into protein sequences by using TBtools [39]. Two methods were used for the identification of TIFY family genes in this work. First, the Hidden Markov Model (HMM) profiles of the TIFY domain (Pfam: PF06200) were downloaded from Pfam (http://pfam.xfam.org/) and used for protein screening in HMMER 3.2.1 (e-value < 0.01) [40]. The first part of the candidate TIFY family proteins was obtained. In addition, BLAST analyses were conducted using 18 Arabidopsis [7] and 36 Brassica rapa [26] TIFY protein sequences as queries on the D134 genome by using the BLASTP program, thus obtaining the second part of the candidate TIFY proteins in B. oleracea. Subsequently, the two candidate sets were merged, the redundant proteins were removed, and their conserved domains were further identified by using NCBI-CDD (https://www.ncbi.nlm.nih.gov/Structure/cdd/wrpsb.cgi) [41]. Finally, cabbage TIFY family genes were obtained from the D134 genome and used for subsequent analysis.

The theoretical isoelectric point $(\mathrm{pI})$ and molecular weight $(\mathrm{Mw})$ of each cabbage TIFY protein were analysed using the 'Compute $\mathrm{pI} / \mathrm{Mw}$ tool' in ExPASy (https://web.expasy.org/compute_pi/). The subcellular locations were predicted using ProtComp 9.0 from Softberry (http://linux1.softberry.com/ berry.phtml) and Plant-mPLoc in Cell-PLoc 2.0 (http://www.csbio.sjtu.edu.cn/bioinf/Cell-PLoc-2/).

\subsection{Chromosomal Location and Tandem Duplication Analysis}

Based on the genomic sequence annotation file provided by the D134 genome database, the chromosomal position of the cabbage TIFY family genes were obtained and drew maps using TBtools. MCscanX software (http://chibba.pgml.uga.edu/mcscan2/) was used to search tandem and duplicated genes. The $\mathrm{Ka} / \mathrm{Ks}$ analysis of the TIFY family genes was also conducted using TBtools.

\subsection{Gene Structure, Conserved Motif, and Phylogenetic Analyses}

To investigate the intron-exon organization of the cabbage TIFY proteins, the coding sequences with corresponding genomic sequences were aligned, and the results were obtained online using the Gene Structure Display Server (GSDS, http://gsds.cbi.pku.edu.cn/index.php) [42]. The conserved motifs in full-length TIFY proteins were identified by MEME (http://meme-suite.org/) [43]. The hylogenetic analysis of B. oleracea, B. rapa, and A. thaliana TIFY proteins was generated by using the Molecular Evolutionary Genetics Analysis software package, Version 6 (MEGA 6) with the neighbour-joining (NJ) algorithm [44]. Bootstrap analysis with 1000 replications was performed to assess group support.

\subsection{Expression Pattern Analysis of Cabbage TIFY Genes Using RNA-Seq Data}

The RNA-seq data of various cabbage tissues (bud, callus, root, stem, leaf, flower, and silique) were downloaded from the DDBJ/EMBL/GenBank Sequence Read Archive (SRA) database (GSE42891) and used for the expression analysis of the cabbage TIFY genes. To determine the expression patterns of JAZ subfamily genes after different pathogen infections, the RNA-seq data sets of cabbage clubroot resistance 
(XG) and susceptible (JF) lines (28 days after P. brassicae inoculation and the control mock-inoculated with sterile water, https://www.ncbi.nlm.nih.gov/sra/SRP144315), and Fusarium wilt resistance (96-100) and susceptible (01-20) lines (0 and 3 days after F. oxysporum inoculation, https://www.ncbi.nlm.nih. gov/bioproject/PRJNA548392) were downloaded from SRA database. The data sets of cabbage black rot resistance (Fuji early) and susceptible (87-534) lines ( 0 and 6 days after X. campestris inoculation) were obtained from relevant research in our laboratory (unpublished). All high-quality reads of each sample that passed the quality control were mapped to the B. oleracea reference genome, and the uniquely mapped reads were used for expression analysis. The fragments per kb per million (FPKM) method was conducted to normalize and calculate the gene expression levels of TIFY genes in different tissues or JAZ subfamily genes after different pathogen inoculations [45]. The negative binomial (NB) distribution test in the DESeq software package (http://bioconductor.org/packages/release/bioc/html/DESeq.html) was used to test the significance of differences (Fold Changes $\geq 2$ and $p$-value $\leq 0.05$ ). The heat maps of hierarchical clustering were constructed in TBtools.

\subsection{Phytohormone Treatment and Quantitative Real-Time PCR Analysis}

The cabbage cultivar 'Zhonggan No. 21' provided by the Cabbage and Broccoli Research Group, the Institute of Vegetables and Flowers, the Chinese Academy of Agricultural Sciences (IVFCAAS), was used for the expression analyses after different phytohormone treatments. In a greenhouse, cabbage seedlings were cultivated at $28^{\circ} \mathrm{C}$ with $14 \mathrm{~h}$ of light/10 h of dark under artificial light until the three-leaf stage. Salicylic acid (SA) and MeJA were dissolved in 100\% ethanol, and ethephon was dissolved in sterile distilled water to suitable concentrations as stock solutions and diluted with sterile distilled water containing Tween $20[0.1 \%(v / v)]$ for foliar spraying. Sterile distilled water containing Tween $20[0.1 \%(v / v)]$ was used as the mock control. The cabbage seedlings were sprayed with $100 \mu \mathrm{M} \mathrm{SA}$, $100 \mu \mathrm{M} \mathrm{MeJA}$ and $500 \mathrm{mg} / \mathrm{L}$ ethephon. There were three repetitions in every treatment, and each repetition consisted of 10 plants. After two hours, the leaf samples of every treatment were taken and frozen in liquid nitrogen and stored at $-80{ }^{\circ} \mathrm{C}$ for RNA extraction.

Total RNA of the leaf samples was extracted using a FastPure Plant Total RNA Isolation Kit (Vazyme Biotech Co., Nanjing, Jiangsu Province, China) following the manufacturer's instructions. The quantity and purity of RNA were estimated using an ND-1000 spectrophotometer (Thermo Fisher Scientific Inc., Wilmington, DE, USA). First-stand cDNAs were synthesized by reverse transcription using HiScript ${ }^{\circledR}$ III RT SuperMix (Vazyme Biotech) following the manufacturer's instructions and diluted to $50 \mathrm{ng} / \mu \mathrm{L}$ for downstream processing. The specific primers of JAZ genes were designed using Premier 6 software. The sequences, amplification length, and locations of each primer have been listed in Table S1, and the specificity of the amplification products was test by agarose gel electrophoresis (Figure S1). Each reaction contained $1.0 \mu \mathrm{L}$ of cDNA, $0.4 \mu \mathrm{L}$ of forward and reverse primer $(10 \mu \mathrm{M})$, $10.0 \mu \mathrm{L}$ of $2 \times$ ChamQ Universal SYBR qPCR Master Mix (Vazyme Biotech), and $8.2 \mu \mathrm{L}$ double-distilled $\mathrm{H}_{2} \mathrm{O}$ in a total reaction volume of $20 \mu \mathrm{L}$ and was conducted in a Bio-Rad CFX96 Real-Time PCR System (Bio-Rad Laboratories, Hercules, CA, USA) with three technical replicates by using hard-shell PCR plates (HSP9601, Bio-Rad Laboratories). Conditions for the reaction were as follows: $95^{\circ} \mathrm{C}$ for $3 \mathrm{~min}$, followed by 45 cycles of $95^{\circ} \mathrm{C}$ for $10 \mathrm{~s}, 60^{\circ} \mathrm{C}$ for $30 \mathrm{~s}$, and $72{ }^{\circ} \mathrm{C}$ for $20 \mathrm{~s}$. The delta-delta $\mathrm{Ct}\left(2^{-\Delta \Delta \mathrm{Ct}}\right)$ algorithm was used to analyse the relative gene expression levels [46,47]. Actin (GenBank accession number XM_013731369.1, Table S1) was used as the internal control to normalize the expression of the target genes. Between phytohormone treated and control samples, statistical analysis to find significant differential expression was determined using a two-tailed Student's $t$-test in Microsoft Office Excel 2017 ( $p$-values $<0.05 \alpha$-level).

\subsection{Subcellular Localization}

The pCAMBIA1300-GFP vector was used for the subcellular localization test and digested with two restriction endonucleases (XbaI and KpnI) to insert the target genes. The CDS sequences of six JAZ genes were amplified with specific primer pairs with homologous arms (Table S2), and the amplification 
products were recovered using the FastPure Gel DNA Extraction Mini Kit (Vazyme Biotech). Through homologous recombination, the six JAZ genes were connected to the pCAMBIA1300-GFP vector by using the ClonExpress ${ }^{\circledR}$ Ultra One Step Cloning Kit (Vazyme Biotech). Then, the recombinant plasmids were transferred into Agrobacterium tumefaciens strain GV3101. The transformed Agrobacterium tumefaciens was cultured for $24 \mathrm{~h}$ at $28{ }^{\circ} \mathrm{C}$ in L-broth supplemented with $50 \mu \mathrm{g} / \mathrm{mL}$ kanamycin, sedimented by centrifugation at $5000 \times g$ for $10 \mathrm{~min}$ at room temperature and resuspended in sterile distilled water containing $10 \mathrm{mM} \mathrm{MgCl}_{2}$ and $150 \mu \mathrm{g} / \mathrm{mL}$ acetosyringone to an optical density (OD600) of 1.0. After standing for $2 \mathrm{~h}$, cells were infiltrated into the abaxial air spaces of Nicotiana benthamiana plants [48]. Forty-eight hours after infiltration, the expression position of the JAZ proteins was observed by a Leica SP8 laser confocal microscope (Leica Microsystems, Inc., Buffalo Grove, IL, USA) using filter blocks to select for spectral emission at $488 \mathrm{~nm}$ (matching the GFP), and the empty vector was used as a control.

\section{Results}

\subsection{Genome-Wide Identification of the TIFY Family Genes in Cabbage}

On the basis of HMMER search results, 34 TIFY proteins were identified in the D134 genome (Table S3) and were taken as the first part of the candidate TIFY proteins. Then, 79 and 89 homologous proteins were obtained according to the BLASTP search using 18 A. thaliana TIFY proteins and $36 B$. rapa TIFY proteins (Table S3), respectively. Subsequently, all the candidate TIFY proteins were merged and scanned using NCBI-CDD for the identification of their conserved domains. Finally, a total of 36 non-redundant TIFY genes were identified in the D134 genome of B. oleracea, including 22 JAZ, 2 PPD, 5 ZML and 7 TIFY proteins. The gene locus IDs of the 36 TIFY genes in the D134 genome are shown in Table 1, and the homologous loci in the 02-12 genome were present simultaneously (4 TIFY genes have no homologous genes in the 02-12 genome). The nucleotide and amino acid sequences of these TIFY genes are summarized in Table S4. The length of these TIFY proteins ranged from 113 (Boc04g01322) to 359 (Boc03g00474) amino acid (aa) residues with an average length of 236 aa. The molecular weight ranged from 11939.03 Da to 38823.97 Da, and the pI values varied from 4.68 to 10.07. Subcellular location prediction showed that all TIFY proteins were predicted in the nucleus.

\subsection{Chromosomal Location and Gene Duplication Analysis of the Cabbage TIFY Genes}

All 36 TIFY genes were assigned to nine chromosomes of B. oleracea (Figure 1), and the distribution of the TIFY genes on each chromosome was uneven. Chromosome 3 contained the largest number of TIFY genes (6 genes), followed by chromosomes 2, 5, 6, and 8, which contained 5 genes. Only one TIFY gene was located on chromosome 7. Based on the chromosomal location and the subfamily classification, the 36 TIFY genes in B. oleracea were renamed (BoJAZ1-BoTIFY7) (Figure 1 and Table 1). 
Table 1. List of the 36 TIFY genes in B. oleracea.

\begin{tabular}{|c|c|c|c|c|c|c|c|c|}
\hline & $\begin{array}{c}\text { Gene } \\
\text { Names }\end{array}$ & Accession No. ${ }^{a}$ & $\begin{array}{l}\text { Homologous } \\
\text { Loci }^{\text {b }}\end{array}$ & $\begin{array}{c}\text { Chromosome } \\
\text { No. }\end{array}$ & $\begin{array}{l}\text { Length } \\
\text { (aa) }\end{array}$ & pI & $\begin{array}{l}\text { MW } \\
\text { (Da) }\end{array}$ & $\begin{array}{c}\text { Localization } \\
\text { Predicted }\end{array}$ \\
\hline 1 & BoJAZ1 & Boc02g02049 & Bol039351 & Chr02 & 241 & 9.30 & 26238.56 & $\mathrm{~N}^{\mathrm{c}}$ \\
\hline 2 & BoJAZ2 & Boc02g02168 & Bol034876 & Chr02 & 158 & 6.16 & 17527.33 & $\mathrm{~N}$ \\
\hline 3 & BoJAZ3 & Boc02g02274 & Bol041431 & Chr02 & 270 & 9.96 & 28913.75 & $\mathrm{~N}$ \\
\hline 4 & BoJAZ4 & Boc02g03465 & Bol036100 & Chr02 & 221 & 4.97 & 23089.94 & $\mathrm{~N}$ \\
\hline 5 & BoJAZ5 & Boc02g03937 & - & Chr02 & 197 & 9.88 & 21860.19 & $\mathrm{~N}$ \\
\hline 6 & BoJAZ6 & Boc03g01228 & Bol008534 & Chr03 & 131 & 9.85 & 14971.78 & $\mathrm{~N}$ \\
\hline 7 & BoJAZ7 & Boc03g06386 & Bol034224 & Chr03 & 196 & 9.95 & 21688.00 & $\mathrm{~N}$ \\
\hline 8 & BoJAZ8 & Boc04g01076 & Bol027372 & Chr04 & 117 & 9.15 & 13127.05 & $\mathrm{~N}$ \\
\hline 9 & BoJAZ9 & Boc05g01466 & Bol026828 & Chr05 & 255 & 9.71 & 27255.92 & $\mathrm{~N}$ \\
\hline 10 & BoJAZ10 & Boc05g02247 & Bol022524 & Chr05 & 133 & 9.62 & 15274.14 & $\mathrm{~N}$ \\
\hline 11 & BoJAZ11 & Boc05g03357 & Bol013829 & Chr05 & 336 & 9.47 & 35762.95 & $\mathrm{~N}$ \\
\hline 12 & BoJAZ12 & Boc06g00449 & Bol026137 & Chr06 & 236 & 9.30 & 26035.25 & $\mathrm{~N}$ \\
\hline 13 & BoJAZ13 & Boc06g00630 & Bol026339 & Chr06 & 272 & 9.47 & 30322.98 & $\mathrm{~N}$ \\
\hline 14 & BoJAZ14 & Boc06g00780 & - & Chr06 & 267 & 9.71 & 28723.60 & $\mathrm{~N}$ \\
\hline 15 & BoJAZ15 & Boc06g01385 & Bol017418 & Chr06 & 232 & 9.03 & 25342.54 & $\mathrm{~N}$ \\
\hline 16 & BoJAZ16 & Boc06g01609 & Bol039922 & Chr06 & 225 & 9.23 & 24461.58 & $\mathrm{~N}$ \\
\hline 17 & BoJAZ17 & Boc08g00802 & Bol009774 & Chr08 & 344 & 9.43 & 38025.74 & $\mathrm{~N}$ \\
\hline 18 & BoJAZ18 & Boc08g00884 & Bol044840 & Chr08 & 276 & 9.43 & 30024.88 & $\mathrm{~N}$ \\
\hline 19 & BoJAZ19 & Boc08g02786 & Bol013163 & Chr08 & 292 & 9.51 & 31837.28 & $\mathrm{~N}$ \\
\hline 20 & BoJAZ20 & Boc08g02867 & Bol029321 & Chr08 & 269 & 8.84 & 29748.45 & $\mathrm{~N}$ \\
\hline 21 & BoJAZ21 & Boc09g03643 & Bol035782 & Chr09 & 203 & 6.85 & 21420.92 & $\mathrm{~N}$ \\
\hline 22 & BoJAZ22 & Boc09g04265 & Bol043451 & Chr09 & 198 & 10.07 & 21998.47 & $\mathrm{~N}$ \\
\hline 23 & BoPPD1 & Boc01g02166 & Bol014725 & Chr01 & 318 & 8.64 & 34439.52 & $\mathrm{~N}$ \\
\hline 24 & BoPPD2 & Boc08g03725 & Bol006854 & Chr08 & 308 & 8.78 & 33583.44 & $\mathrm{~N}$ \\
\hline 25 & BoZML1 & Boc01g01504 & Bol009539 & Chr01 & 305 & 6.05 & 33079.78 & $\mathrm{~N}$ \\
\hline 26 & BoZML2 & Boc01g03057 & Bol018802 & Chr01 & 289 & 6.26 & 31531.93 & $\mathrm{~N}$ \\
\hline 27 & BoZML3 & Boc03g00258 & - & Chr03 & 305 & 6.11 & 33290.86 & $\mathrm{~N}$ \\
\hline 28 & BoZML4 & Boc05g02989 & Bol038395 & Chr05 & 291 & 6.13 & 31792.40 & $\mathrm{~N}$ \\
\hline 29 & BoZML5 & Boc07g00805 & Bol042168 & Chr07 & 260 & 5.74 & 28003.17 & $\mathrm{~N}$ \\
\hline 30 & BoTIFY1 & Boc01g00534 & Bol017893 & Chr01 & 345 & 9.51 & 37246.04 & $\mathrm{~N}$ \\
\hline 31 & BoTIFY2 & Boc03g00474 & Bol017492 & Chr03 & 359 & 8.49 & 38823.97 & $\mathrm{~N}$ \\
\hline 32 & BoTIFY3 & Boc03g01224 & Bol016130 & Chr03 & 122 & 5.06 & 13390.66 & $\mathrm{~N}$ \\
\hline 33 & BoTIFY4 & Boc03g05199 & Bol036968 & Chr03 & 114 & 8.97 & 13033.89 & $\mathrm{~N}$ \\
\hline 34 & BoTIFY5 & Boc04g01322 & Bol014138 & Chr04 & 113 & 4.68 & 11939.03 & $\mathrm{~N}$ \\
\hline 35 & BoTIFY6 & Boc04g04270 & Bol037853 & Chr04 & 122 & 9.10 & 13653.55 & $\mathrm{~N}$ \\
\hline 36 & BoTIFY7 & Boc05g01338 & - & Chr05 & 182 & 8.59 & 20042.45 & $\mathrm{~N}$ \\
\hline
\end{tabular}

a The gene locus ID in D134 genome; ${ }^{b}$ The homologous gene locus in 02-12 genome, '-' represent no homologous gene; ${ }^{\mathrm{c}}$ Nucleus.

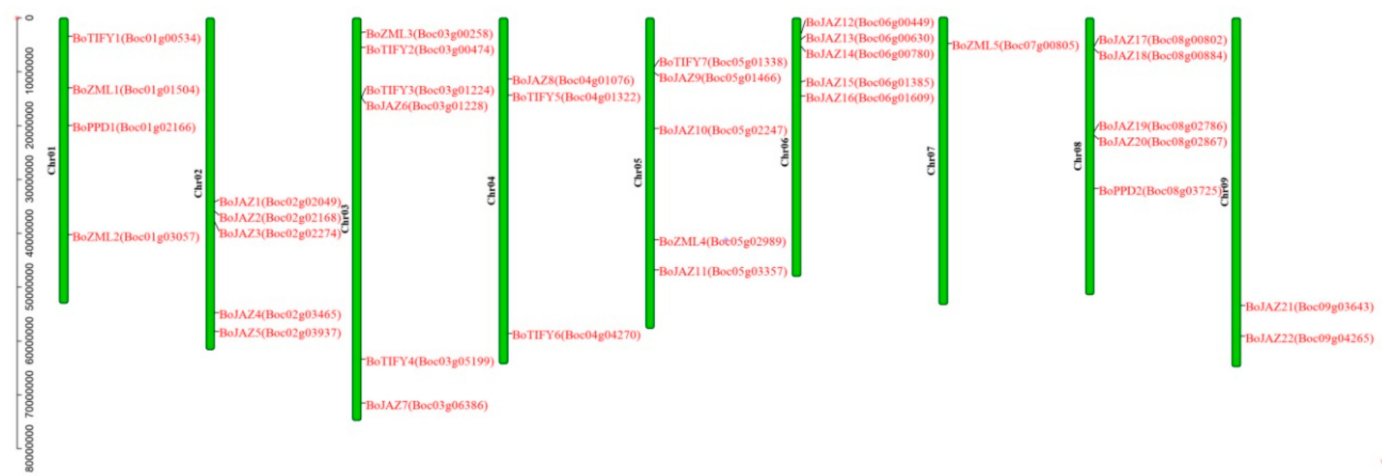

Figure 1. Distribution of TIFY genes on B. oleracea chromosomes. Thirty-six TIFY genes (rename and gene locus ID) are shown on the right of each chromosome. Gene positions and chromosome size can be measured using the scale on the left of the figure in mega bases (bp).

Gene duplication is one of the most important characteristics of plant genomic structure, which usually contributes to the expansion of gene families. Due to the importance of gene duplication in the evolution of gene families in plants, the duplication patterns of 36 putative TIFY genes were analysed in the cabbage genome. A total of 26 duplicated gene pairs were identified by whole genome duplication 
(WGD) (Figure 2). The ratios of $\mathrm{Ka} / \mathrm{Ks}$ can be used as an indicator for the selection pressure of a gene during evolution. The values of all the duplicated TIFY gene pairs were less than 1 in $B$. oleracea (Table 2), indicating that the TIFY genes primarily evolved under the influence of purifying selection.

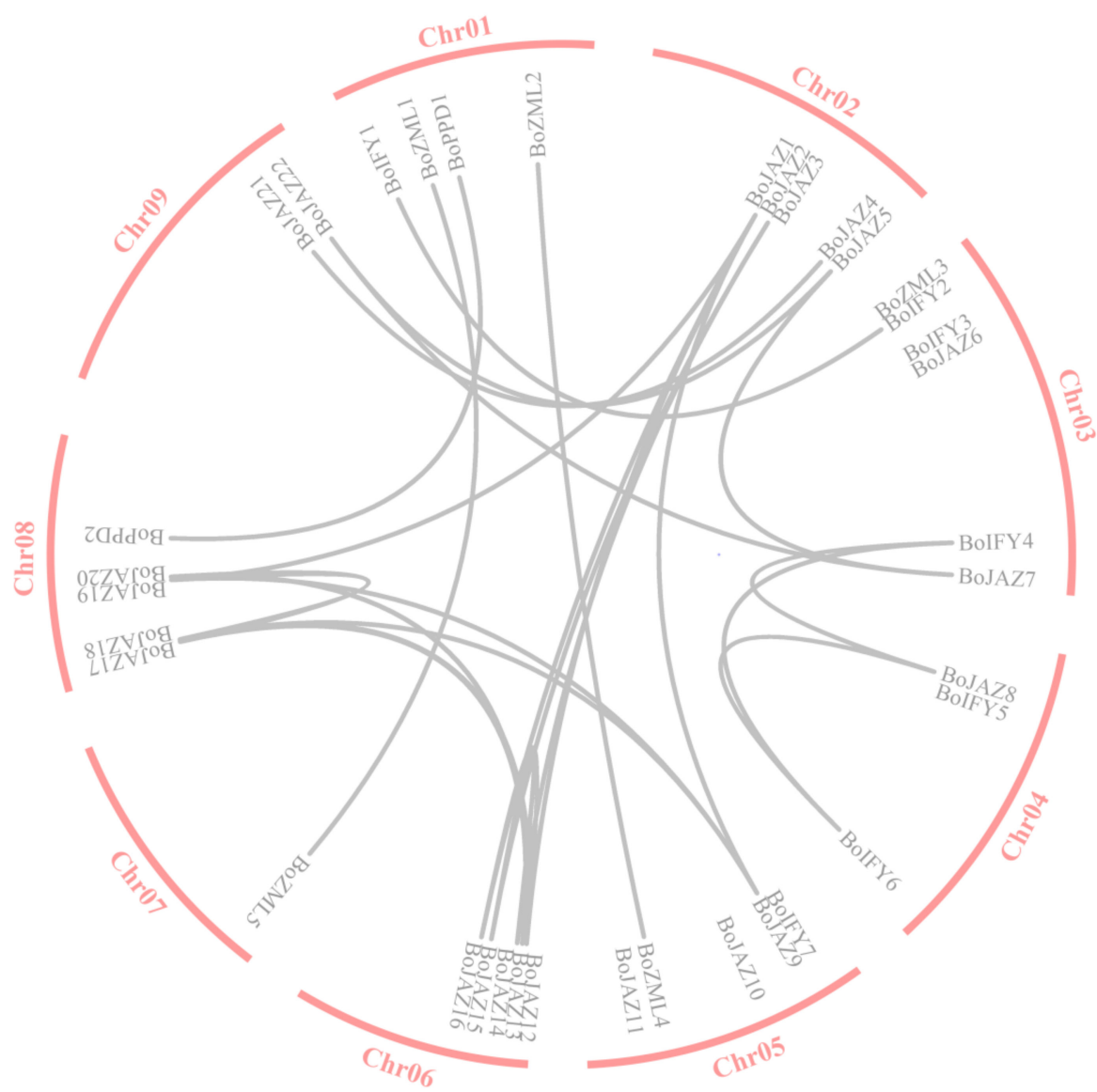

Figure 2. Duplication of the TIFY family genes in B. oleracea. The duplicated gene pairs are joined by grey lines.

\subsection{Gene Structure, Conserved Motif, and Phylogenetic Analysis of the Cabbage TIFY Genes}

The divergence of the exon-intron organization played a critical role in the evolution of multiple gene families. To study the structural diversity of the cabbage TIFY genes, untranslated regions (UTRs), exons and intron organization of each TIFY gene were investigated (Figure 3A). The majority of the TIFY genes contained more than two exons. BoPPD1 and BoPPD2 have the largest number of exons at 8, whereas two TIFY subfamily genes (BoTIFY4 and BoTIFY5) had only one exon and no intron. The length and position of exons and introns of cabbage TIFY genes were varied. In addition, the conserved motifs were examined using the MEME motif search tool, five consensus motifs (tify, Jas, PPD, CCT, ZnF_GATA) were detected in the cabbage TIFY genes, and the distribution of these conserved motifs was further analysed (Figure 3B). All 36 cabbage TIFY genes contained the tify motif, and these genes in the same subfamily have consistent motifs. Seven TIFY subfamily genes only contained the tify motif, while five ZML subfamily genes had the extra CCT and ZnF_GATA motifs, 2 PPD genes had the PPD motif, and the largest JAZ subfamily had 22 members with the C-terminal Jas motif. 
Table 2. Estimated $\mathrm{Ka} / \mathrm{Ks}$ ratios of the duplicated TIFY genes in B. oleracea.

\begin{tabular}{|c|c|c|c|c|c|c|c|}
\hline No. & Paralogous Pairs & $\mathrm{Ka}^{\mathrm{a}}$ & $\mathrm{Ks}^{\mathrm{b}}$ & $\mathrm{Ka} / \mathrm{Ks}$ & $\begin{array}{c}\text { Effective } \\
\text { Length (bp) }\end{array}$ & $\begin{array}{l}\text { Average } \\
\text { S-Sites }{ }^{c}\end{array}$ & $\begin{array}{l}\text { Average } \\
\text { N-Sites }{ }^{d}\end{array}$ \\
\hline 1 & BoJAZ1/BoJAZ12 & 0.136239503 & 0.466279810 & 0.292184007 & 681 & 157.92 & 523.08 \\
\hline 2 & BoJAZ1/BoJAZ9 & 0.288117541 & 0.801855456 & 0.359313564 & 705 & 167.17 & 537.83 \\
\hline 3 & BoJAZ1/BoJAZ19 & 0.289411753 & 0.901233386 & 0.321128531 & 702 & 166.83 & 535.17 \\
\hline 4 & BoJAZ3/BoJAZ14 & 0.090640706 & 0.237924642 & 0.380963929 & 786 & 184.75 & 601.25 \\
\hline 5 & BoJAZ3/BoJAZ15 & 0.129965792 & 0.277005584 & 0.469181128 & 669 & 152.92 & 516.08 \\
\hline 6 & BoJAZ4/BoJAZ21 & 0.116023822 & 0.322633719 & 0.359614682 & 600 & 151.92 & 448.08 \\
\hline 7 & BoJAZ5/BoJAZ7 & 0.116977545 & 0.248486377 & 0.470760395 & 585 & 141.83 & 443.17 \\
\hline 8 & BoJAZ5/BoJAZ22 & 0.077915489 & 0.387530995 & 0.201056147 & 588 & 142.08 & 445.92 \\
\hline 9 & BoTIFY4/BoTIFY6 & 0.125567214 & 0.275793585 & 0.455294180 & 318 & 71.50 & 246.50 \\
\hline 10 & BoTIFY4/BoJAZ8 & 0.128368343 & 0.326326040 & 0.393374500 & 303 & 69.92 & 233.08 \\
\hline 11 & BoJAZ7/BoJAZ22 & 0.120085286 & 0.276642241 & 0.434081524 & 585 & 141.92 & 443.08 \\
\hline 12 & BoJAZ8/BoTIFY6 & 0.116309928 & 0.170137651 & 0.683622511 & 348 & 78.83 & 269.17 \\
\hline 13 & BoJAZ9/BoJAZ12 & 0.291213635 & 0.704555605 & 0.413329527 & 684 & 157.42 & 526.58 \\
\hline 14 & BoJAZ9/BoJAZ18 & 0.096000993 & 0.259398924 & 0.370090173 & 756 & 177.08 & 578.92 \\
\hline 15 & BoJAZ9/BoJAZ19 & 0.084821096 & 0.284963797 & 0.297655691 & 741 & 175.75 & 565.25 \\
\hline 16 & BoJAZ12/BoJAZ16 & 0.185531899 & 0.474852727 & 0.390714611 & 621 & 138.33 & 482.67 \\
\hline 17 & BoJAZ12/BoJAZ18 & 0.269450506 & 0.922697085 & 0.292024881 & 693 & 158.08 & 534.92 \\
\hline 18 & BoJAZ13/BoJAZ17 & 0.270636401 & 1.000601128 & 0.270473812 & 795 & 179.50 & 615.50 \\
\hline 19 & BoJAZ13/BoJAZ20 & 0.254382445 & 1.085309439 & 0.234387020 & 774 & 173.33 & 600.67 \\
\hline 20 & BoJAZ14/BoJAZ15 & 0.157843966 & 0.338195612 & 0.466723875 & 657 & 150.00 & 507.00 \\
\hline 21 & BoJAZ17/BoJAZ20 & 0.130211313 & 0.385659245 & 0.337633065 & 804 & 180.75 & 623.25 \\
\hline 22 & BoJAZ18/BoJAZ19 & 0.082922850 & 0.304787220 & 0.272068001 & 774 & 181.67 & 592.33 \\
\hline 23 & BoTIFY1/BoTIFY2 & 0.094462148 & 0.272162609 & 0.347079816 & 1014 & 236.58 & 777.42 \\
\hline 24 & BoZML1/BoZML5 & 0.112145386 & 0.405464237 & 0.276585148 & 774 & 183.58 & 590.42 \\
\hline 25 & BoZML2/BoZML4 & 0.059319442 & 0.309010440 & 0.191965817 & 849 & 191.50 & 657.50 \\
\hline 26 & BoPPD1/BoPPD2 & 0.081285177 & 0.335838405 & 0.242036575 & 897 & 211.17 & 685.83 \\
\hline
\end{tabular}

${ }^{a}$ Non-synonymous substitution rate; ${ }^{b}$ Synonymous substitution rate; ${ }^{c}$ The average number of synonymous sites; ${ }^{d}$ The average number of non-synonymous sites.

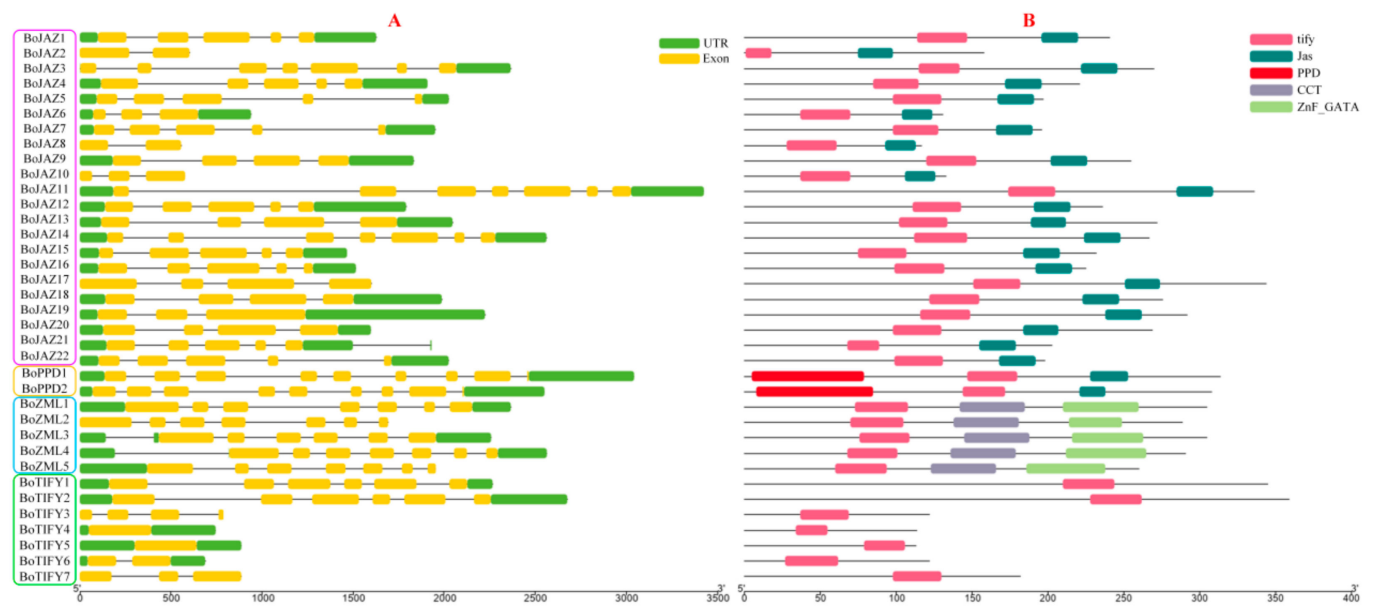

Figure 3. Gene structures (A) and motifs (B) of 36 TIFY genes identified in B. oleracea. UTRs and exons are represented by green and yellow boxes respectively, and introns are represented by grey lines, the length of gene structures can be measured using the scale on the lower in mega bases (bp) (A). Boxes with different colors indicate conserved motifs, and the length of motifs in each protein is shown proportionally and can be measured using the scale on the lower in amino acids (aa) (B).

Based on the amino acid sequences of full-length TIFY proteins in A. thaliana (18) [7], B. oleracea (36) and B. rapa (36) [26], the phylogenetic tree was constructed using the neighbour-joining method in MEGA 6 software. The 91 TIFY proteins were grouped into ten clades (groups 1-10 with different background colours) (Figure 4). Among these clades, group 5 was formed with 13 ZML proteins (3 of A. thaliana, 5 of B. rapa, 5 of B. oleracea), and six PPD proteins (2 of A. thaliana, 2 of B. rapa, 2 of B. oleracea) were gathered together in group 6. Groups 1,3, 7, and 9 were four JAZ subfamily clades, including 14 (2 of A. thaliana, 6 of B. rapa, 6 of B. oleracea), 6 (2 of A. thaliana, 2 of B. rapa, 2 of B. oleracea), 
12 (3 of A. thaliana, 5 of B. rapa, 4 of B. oleracea) and 5 (1 of A. thaliana, 2 of B. rapa, 2 of B. oleracea) JAZ proteins, respectively. Groups 4 and 8 were two TIFY subfamily branches, including 2 ( 1 of $B$. rapa, 1 of B. oleracea) and 5 (1 of A. thaliana, 2 of B. rapa, 2 of B. oleracea) TIFY proteins, respectively. However, group 2 and group 10 were two mixed branches, containing both JAZ and TIFY subfamily members. In group 2, there were $11 \mathrm{JAZ}$ subfamily proteins ( 2 of $A$. thaliana, 5 of B. rapa, 4 of B. oleracea) and 1 cabbage TIFY subfamily protein (BoTIFY7). In group 10, there were $8 \mathrm{JAZ}$ subfamily proteins (2 of $A$. thaliana, 2 of B. rapa, 4 of B. oleracea) and 7 TIFY subfamily proteins ( 4 of B. rapa, 3 of B. oleracea). The same protein number and similar phylogenetic classification of TIFY proteins in B. oleracea and B. rapa may reveal the parallel evolutionary relationship of this family between the two species [49].

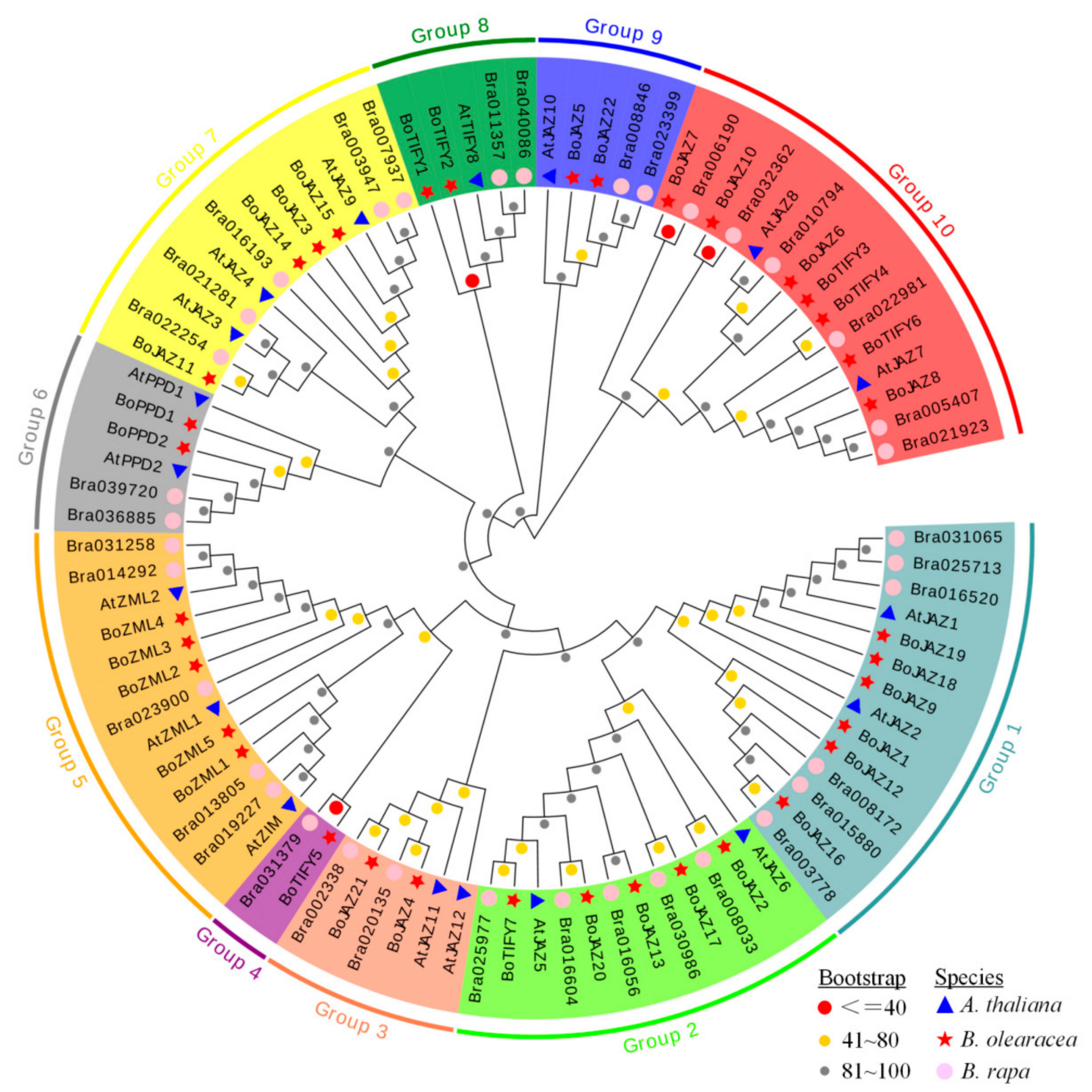

Figure 4. Phylogenetic tree of TIFY genes from B. olearacea, B. rapa, and A. thaliana. The proteins from each species are labeled with different graphics and colors (red star: B. olearacea, pink circle: B. rapa, blue triangle: A. thaliana). The ten groups with different colors represent ten clades. The circles with different colors at the nodes represent bootstrap percentage values (grey: 0-40, yellow: 41-80, red: 81-100) from 1000 replications.

\subsection{Expression Patterns of the Cabbage TIFY Family Genes in Various Tissues}

To explore the expression pattern of the TIFY family genes, RNA-seq data from seven cabbage tissues (root, stem, leaf, bud, flower, callus, and silique) were used (Figure 5 and Table S2). Because these RNA-seq data were obtained from the 02-12 transcriptome sequence data, the four TIFY genes (BoJAZ5, BoJAZ14, BoZML3, BoTIFY7) without homologous loci in the 02-12 genome were not analysed. Lower expression levels of ten JAZ genes in three clades (BoJAZ6/BoJAZ8/BoJAZ10, 
BoJAZ2/BoJAZ13/BoJAZ17/BoJAZ20, BoJAZ9/BoJAZ18/BoJAZ19) were observed in leaves, buds, flowers, and siliques, while these JAZ genes showed the highest expression level in roots. BoJAZ3 and BoJAZ7 were in the same clade with BoJAZ15, BoJAZ17, and BoJAZ22, while the first two had the highest expression in buds and the last three in roots. In addition, BoJAZ1, BoJAZ12, and BoJAZ16 in another clade were highly expressed in silique. For TIFY subfamily genes, the expression levels were diverse in different tissues. Three TIFY subfamily genes (BoTIFY3, BoTIFY4, BoTIFY6) in one clade showed lower expression levels in leaves, buds, flowers, and siliques, while BoTIFY1, BoTIFY2 and BoTIFY5 in another clade were different. Two PPD genes (BoPPD1 and BoPPD2) showed the highest expression in leaves, indicating that they may be involved in the coordination of leaf growth like AtPPD1 and AtPPD2 in A. thaliana [14]. In addition, the four cabbage ZML genes may perform similar biological functions with relatively consistent expression levels in all tissues.

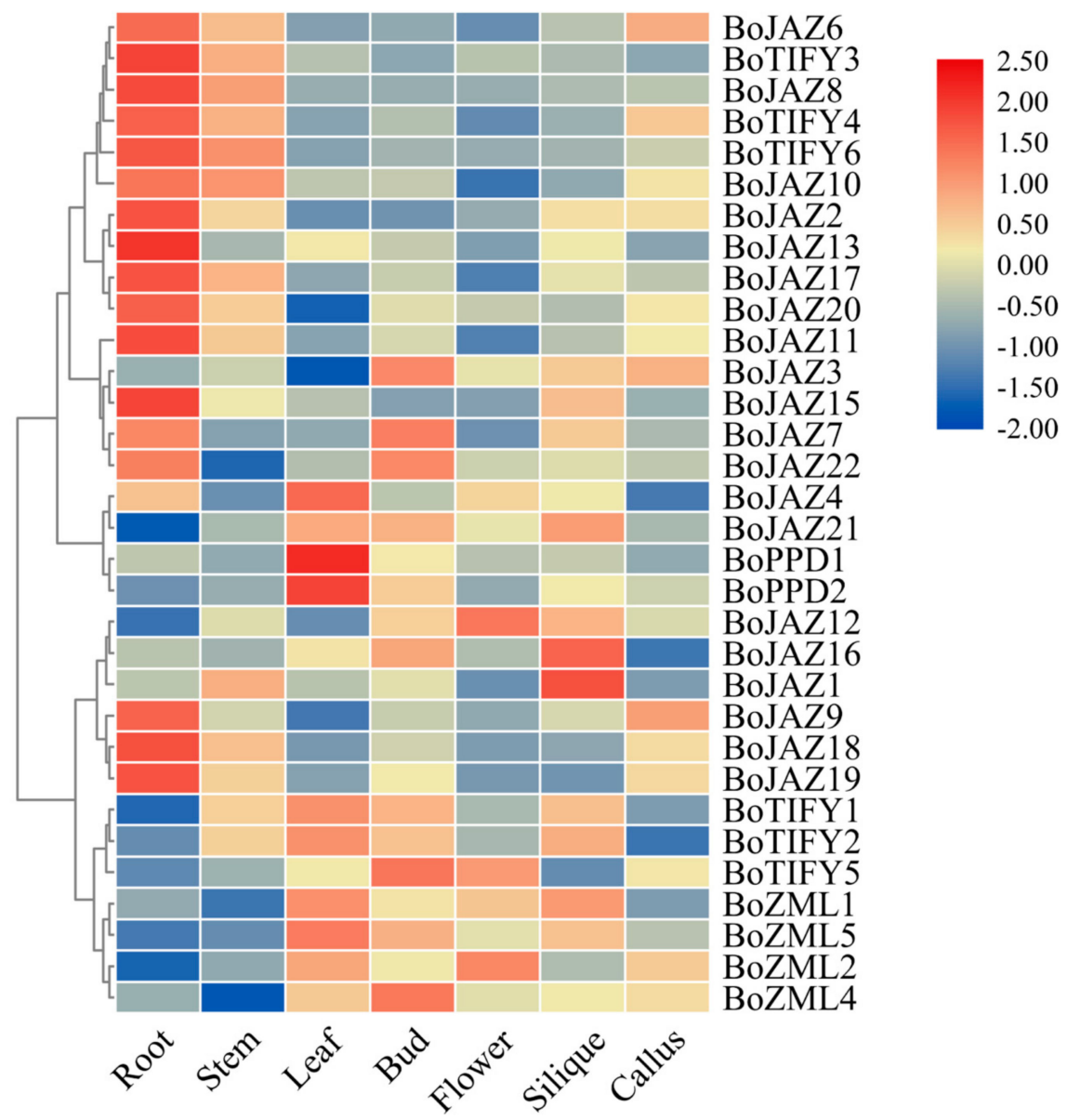

Figure 5. Heat map representation and hierarchical clustering of cabbage TIFY gene expression levels across roots, callus, siliques, stems, leaves, buds, and flowers. $\log _{2}$ transformed values were used to generate the color-coded heatmap, and the color scale with red and blue represent high and low values, respectively, color scale from -2.0 to 2.5 .

\subsection{Expression Profiles of the Cabbage JAZ Genes induced by Different Pathogen Infection}

Jasmonates and related signalling compounds regulate a wide range of biological processes in plants, not only sexual reproduction and development but also host immunity [20,22]. JAZ proteins act as key repressors of JA signalling linking COI1 and downstream transcription factors, suggesting that these proteins may also play key roles in plant defense responses [21,50]. To investigate the expression patterns of cabbage JAZ genes after pathogen infection in both resistant and susceptible materials, RNA-seq data sets of cabbage clubroot, Fusarium wilt, and black rot were used to explore 
their expression differences. Thirteen JAZ genes were significantly upregulated in the cabbage clubroot-resistant line after P. brassicae inoculation, especially BoJAZ10 (Figure 6A and Table S5). For the susceptible line, in addition to BoJAZ4 and BoJAZ21, other JAZ genes were all downregulated to various degrees, and twelve of them were significantly downregulated, especially BoJAZ15 (Figure 6A and Table S5). The opposite responses of JAZ genes between cabbage clubroot-resistant line and susceptible line indicate that JAZ genes may play an important role in the resistant reaction of cabbage to $P$. brassicae. Similar to the reaction of the cabbage-resistant line for P. brassicae, sixteen JAZ genes in the cabbage Fusarium wilt-resistant line were upregulated after F. oxysporum inoculation, and ten of them were significantly upregulated (Figure 6B and Table S5). However, BoJAZ12 and ten other JAZ genes were also upregulated in the susceptible line. After the inoculation of $X$. campestris, a bacterium causing black rot of cabbage, we also found that many JAZ genes were upregulated in both the resistant line and susceptible line (Figure 6C and Table S5). Although many JAZ genes were induced whether inoculated with P. brassicae, F. oxysporum or X. campestris, the specific JAZ genes were different among them. For example, BoJAZ15 and BoJAZ16 were downregulated after F. oxysporum inoculation but upregulated after $X$. campestris inoculation. It was suggested that the mechanism of these JAZ members responding to different pathogen infections were different and highly complex, and both redundancy and antagonism were observed.
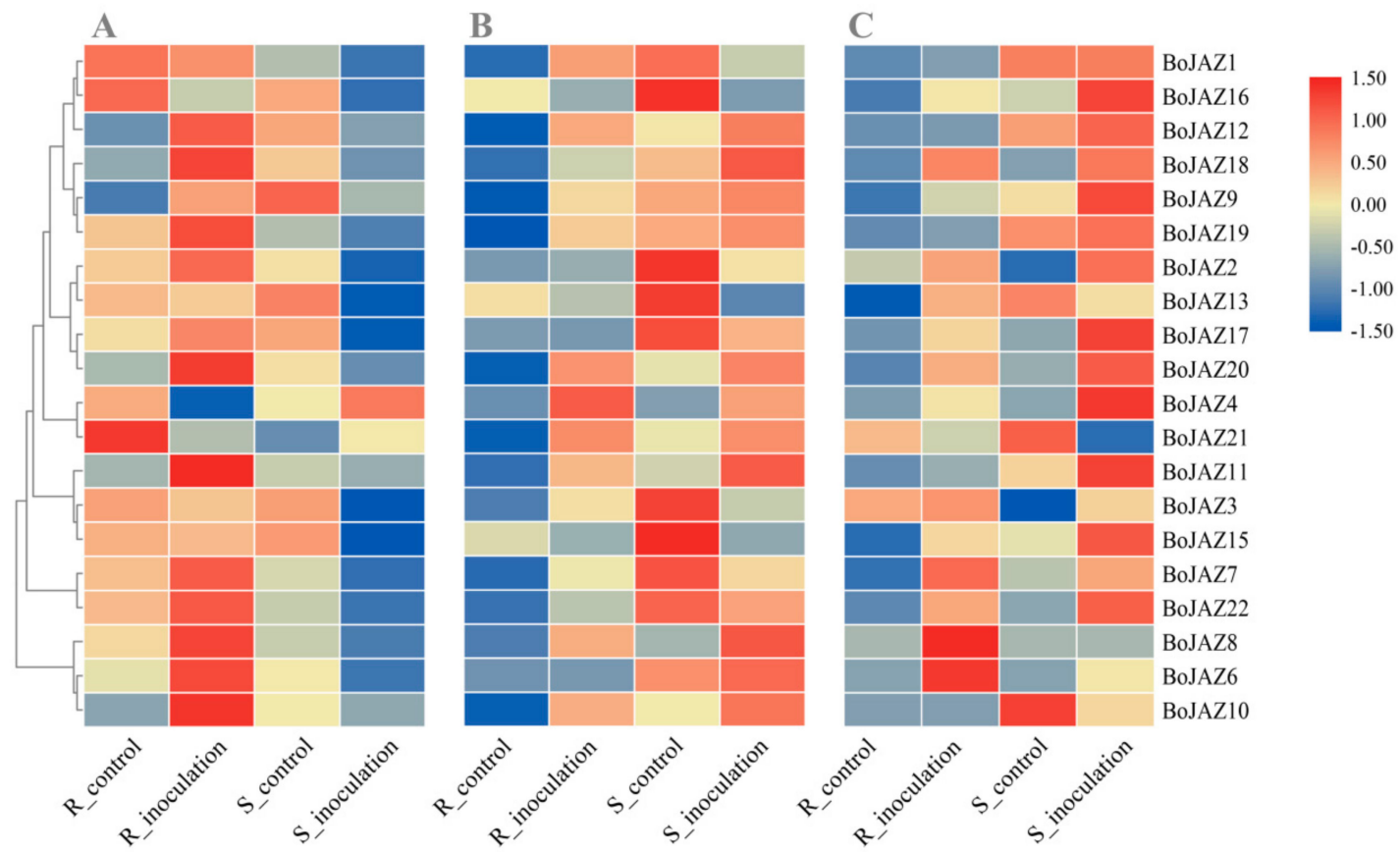

Figure 6. Heat map of cabbage JAZ genes suffering from P. brassicae, F. oxysporum, and X. campestris. (A) expression profile of JAZs after P. brassicae inoculation; (B) expression profile of JAZs F. oxysporum inoculation; (C) expression profile of JAZs after X. campestris inoculation; R: resistant line, S: susceptible line. Log2 transformed values were used to generate the color-coded heatmap, and the color scale with red and blue represent high and low values, respectively, color scale from -1.5 to 1.5.

\subsection{Expression Patterns of the Cabbage JAZ Genes after Exogenous Phytohormone Treatment}

Recent studies have revealed that JAZ proteins may play a role in the regulation of diverse phytohormone signalling pathways involved in defence and plant growth [51,52]. In this work, qRT-PCR was conducted to evaluate the responses of cabbage JAZ genes after different phytohormone (JA, SA, ET) treatments. After treatment with MeJA, the transcription of all JAZ genes in cabbage seedlings was induced compared to the control (Figure 7), which was consistent with the results in A. thaliana and B. rapa [21,26]. Among the JAZ genes, fifteen were significantly upregulated, 
and six (BoJAZ6, BoJAZ8, BoJAZ15, BoJAZ16, BoJAZ18, and BoJAZ20) were upregulated more than 10-fold, while BoJAZ11 was upregulated less than twofold and not significantly, showing the different responses of these JAZ genes to MeJA. In response to SA, we found that most cabbage JAZ genes were downregulated, and five JAZ genes (BoJAZ5, BoJAZ6, BoJAZ7, BoJAZ11, and BoJAZ20) were upregulated. Among these genes, BoJAZ6 and BoJAZ10 had the highest and lowest expression levels, respectively. Similar cases also occurred after ethylene treatment. In addition, we found specific $B o J A Z 6$, which was concurrently induced by the three phytohormones.

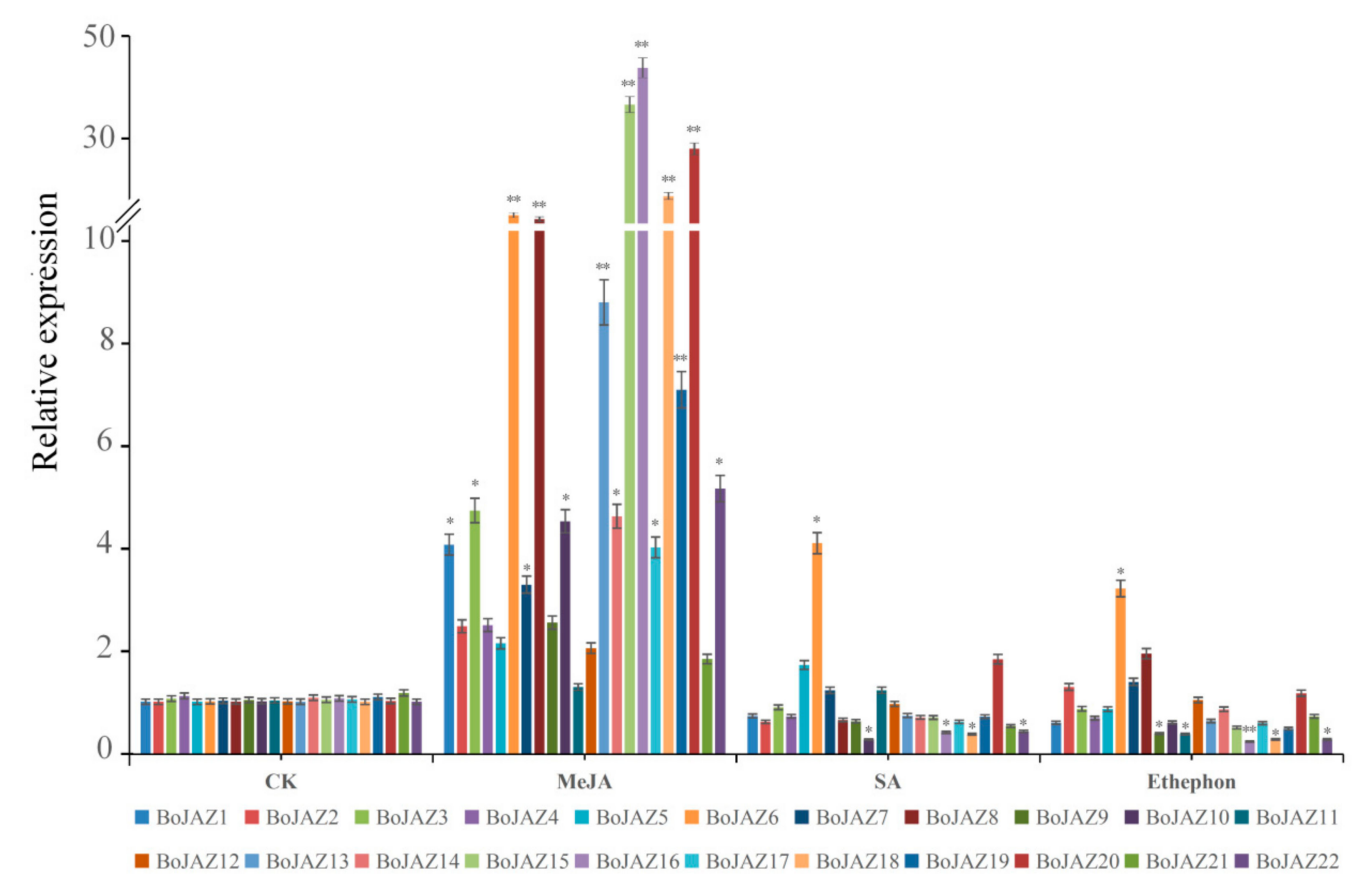

Figure 7. The relative expression of cabbage JAZ genes in the control and exogenous MeJA, SA, and ethylene treatments. Relative expression of JAZ genes was analysed by quantitative real-time qPCR using cabbage actin as a control. Error bars indicate standard deviation, and asterisks indicate significant differences between the control and phytohormone treatment, ${ }^{*} p<0.05,{ }^{* *} p<0.01$.

\subsection{Subcellular Localization of Cabbage JAZ Genes}

Based on the results of the phylogenetic analyses of 91 TIFY genes (Figure 4), twenty-two cabbage JAZ subfamily genes were grouped into six clades $(1,2,3,7,9,10)$, and all of them were predicted to be located in the nucleus by using ProtComp and Plant-mPLoc (Table 1). We recombined the pCAMBIA1300-GFP vector with the CDS sequences of six cabbage JAZ genes (BoJAZ1, BoJAZ2, BoJAZ3, $B o J A Z 4, B o J A Z 5$, and $B o J A Z 6$ ), which participated in the six clades of the phylogenetic tree. The GFP signal of all six JAZ-GFP fusion proteins was observed exclusively in the nucleus (Figure 8), which was consistent with the prediction results. 


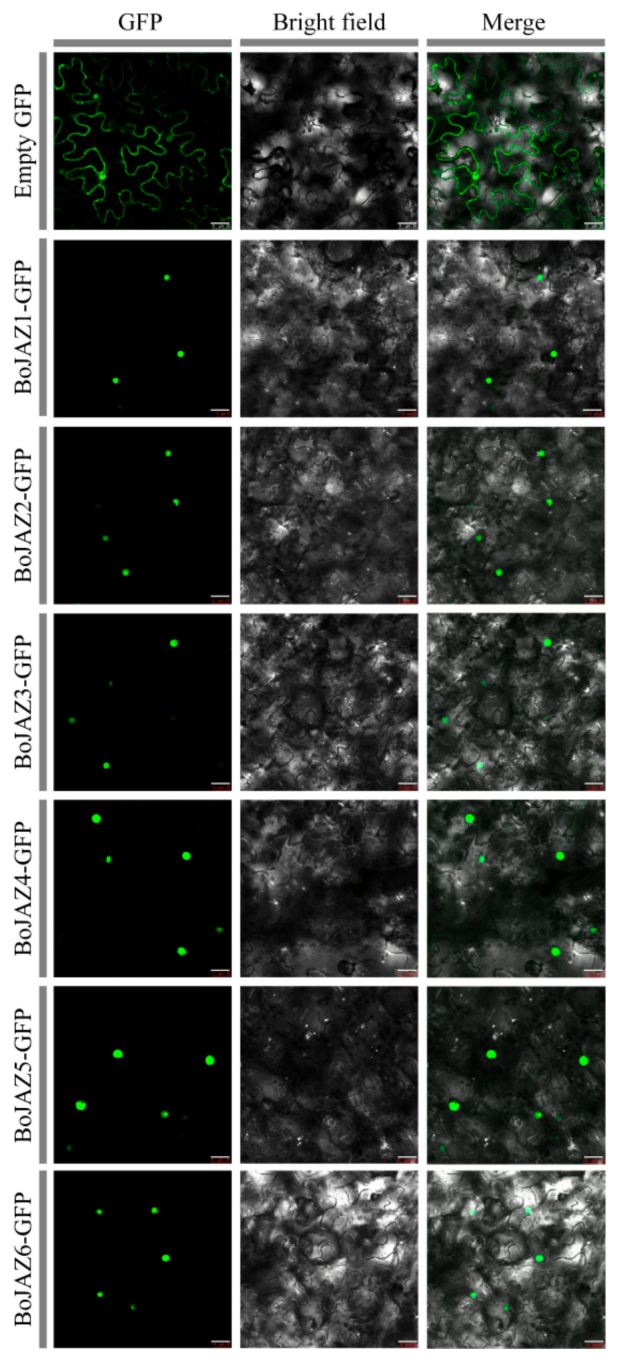

Figure 8. Transient expression of BoJAZ-GFP fusion proteins in tobacco cells. Bars, $25 \mu \mathrm{m}$.

\section{Discussion}

Arabidopsis thaliana, one member of the Brassicaceae family, was the first genome sequenced [53]. Subsequently, the whole-genome sequences for other Brassicaceae members were completed, such as Arabidopsis lyrate, Brassica oleracea, Brassica rapa, Brassica napus, Camelina sativa, and Raphanus sativus [54]. The availability of these genomes improved our understanding of phylogenetic relationships in the Brassicaceae family and laid a solid foundation for genome-wide gene identification and functional research $[55,56]$. Even in closely related species, the genome structure, size and copy number were vary considerably [57], and the variations were restricted to repetitive sequences and affected specific gene families involved in different plant physiological processes [58]. Members of the TIFY family have been demonstrated to be putative TFs with various responsiveness in plant development and defense $[59,60]$. Hence, we performed genome-wide identification and expression profiling analysis of the TIFY gene family in Brassica oleracea. A total of 36 TIFY family genes were identified in the D134 genome of B. oleracea and included 22 JAZ, 2 PPD, 5 ZML and 7 TIFY subfamily genes. The total number of TIFY genes and the number of the four subfamily genes in $B$. oleracea were the same as those in B. rapa. This result indicated that the evolution rate of the TIFY genes was similar between the two Brassica species.

Gene duplication, including tandem, segmental, and whole genome duplication, plays an important role in the evolution of various species and contributes to the expansion of gene families [61,62]. Previous whole genome identification found a total of 1825 gene clusters containing 4365 tandemly duplicated in 
B. oleracea, and there were similar numbers in B. rapa (5181) and A. thaliana (4170) [34]. In this study, 26 duplicated gene pairs of 36 TIFY genes in B. oleracea caused by WGD were identified. WGD is one of the important mechanisms of species evolution, producing new genomic regions and making it more complex and diverse [63]. Similar conditions have been found in other species, such as 19 of the 24 poplar TIFY genes produced by WGD [64]. The large proportion of duplicated genes in the TIFY family revealed that WGD made a large contribution to the generation of the TIFY gene family. Although the duplicated TIFY genes may have a common ancestor, their functions and expression patterns were complex, since duplicated genes can undergo substantial changes in their structures and/or regulatory mechanisms to assume novel roles [62,63]. For example, in the duplicated gene pair of BoJAZ1/BoJAZ19 identified in this work, BoJAZ1 has five exons with 241 amino acid residues, while BoJAZ19 has three exons with 292 amino acid residues, and their expression levels were different in various cabbage tissues.

JA regulates large-scale changes in gene expression to exert its many effects in such processes as plant defense response, cell division, photomorphogenesis, and sexual reproduction [65,66]. JAZ proteins repress the activity of transcription factors that execute responses to JA [20-22]. JAZ proteins contain two highly conserved sequence regions: the C-terminal Jas domain, which plays a key role in destabilizing the repressor for the response of JA-Ile, and the ZIM/TIFY domain, which mediates homo- and heteromeric interactions between most JAZs [7,67]. However, a non-TIFY JAZ protein (JAZ13, encoded by $A t 3 g 22275$ ) was also demonstrated as a functional repressor of JA-mediated responses in Arabidopsis [68]. In this work, $22 \mathrm{JAZ}$ genes were identified in B. oleracea, with the largest number being observed among the four TIFY subfamilies, and there were higher expression levels of most JAZ genes in cabbage root compared with other tissues. In view of the important role of JA in regulating root growth [69], we deduced that JAZ proteins may play a key role in this process and other root-related traits.

Phytohormone signalling networks are extensively involved in the process of plant interactions with pests and pathogens, and numerous studies have shown that JAZ targets appear to be mainly TFs associated with hormone regulation, revealing the crosstalk between JA and other plant hormones to some degree [70]. Salicylic acid (SA) is the major signalling molecule associated with the hypersensitive response (HR) and implicated in plant resistance to (hemi)biotrophic pathogens [71], while defenses against necrotrophic pathogens have been linked to JA and ethylene (ET) signalling [72,73]. However, antagonistic relationships between SA and the JA/ET pathway were most often reported [74]. EIN3 and EIL1, two nuclear transcription factors that initiate downstream transcriptional cascades for ET responses, are capable of interacting with JAZ proteins [75]. Meanwhile, EIN3 and EIL1 repressed SID2, a gene encoding an isochorismate synthase required for SA biosynthesis [76]. RGA, a DELLA protein involved in the regulation of gibberellin (GA) signalling, interacted with JAZ proteins to compete for the JAZ-MYC2 interaction, and the 'relief of suppression' model was built [76-79]. In addition, SA-inducible genes were not constitutively expressed in the quadruple-DELLA mutant, indicating that DELLA has a negative effect on SA signalling [80]. In this study, most cabbage JAZ genes were upregulated after MeJA treatment, and different expression levels were also found after SA or ethylene treatment, particularly BoJAZ6. It was indicated that the JAZ genes can respond to JA, SA and ET signalling simultaneously. These results further proved that JAZ proteins may play a key role in the crosstalk among different phytohormones, especially JA, SA and ET.

Since phytohormones play a key role in signal transduction when plants encounter pathogens, we investigated the expression of the JAZ genes in cabbage-resistant and cabbage-susceptible lines after pathogen inoculation. We found that many cabbage JAZ genes were induced after inoculation with both fungal and bacterial diseases. The most obvious response was the cabbage response to P. brassicae; thirteen JAZ genes were upregulated in the resistant line, and eighteen JAZ genes were downregulated in the susceptible line. The role of SA and JA signalling in the resistance response to biotrophic clubroot has been investigated. In A. thaliana, through the expression analysis of SA- and JA-responsive genes, the determination of SA and JA levels and exogenous phytohormone application, both SA and JA pathways were found to contribute to the inhibition of clubroot development [81]. In 
addition, glucosinolates (GSLs) play roles in plant defense response against microbial pathogens [82]. In Chinese cabbage, the increased content of JA has been proven to mediate the accumulation of aliphatic GSLs and is involved in clubroot during the secondary infection stage [83]. In view of the role of JA in the development of clubroot and the higher expression levels of cabbage JAZ genes in root tissue, and the distinct responses of JAZ genes between cabbage clubroot-resistant and susceptible lines after P. brassicae inoculation, functional studies of cabbage JAZ genes for clubroot resistance are warranted. However, the activation of JAZ genes was also observed both in cabbage resistant and susceptible lines after F. oxysporum and X. campestris inoculation, suggesting that the mechanism by which JAZ genes participate in cabbage disease resistance may be complex and diverse, and there may be functional redundancy and antagonism among them.

\section{Conclusions}

In summary, 36 TIFY genes (22 JAZ, 2 PPD, 5 ZML and 7 TIFY) were identified in the B. oleracea genome through genome-wide analysis. The number and length of exons and introns of these TIFY genes were varied, and the conserved motifs of these TIFY genes were consistent in the same subfamily. The expression of these TIFY genes was organ-specific, and a larger number of JAZ genes were activated after different pathogen infections and MeJA treatment. These results presented in this report lay the foundation for further functional characterization of TIFY genes, and improve our understanding of the JAZ genes in plant development and disease resistance through the JA signalling pathway.

Supplementary Materials: The following are available online at http://www.mdpi.com/2073-4425/11/2/127/s1, Table S1: Sequences of 22 specific primer pairs used for qRT-PCR after phytohormone treatment. Table S2: Sequences of six specific primer pairs used for vector construction with homologous arms. Table S3: HMMER and BLASTP search results of the TIFY gene family in B. oleracea. Table S4: Nucleotide and amino acid sequences of 36 TIFY genes in B. oleracea. Table S5: Expression levels of the TIFY genes in different tissues and JAZ genes after pathogen inoculation. Figure S1: Specificity of the qRT-PCR amplifications of JAZ genes.

Author Contributions: Conceptualization, X.L. and C.Z.; Formal analysis, X.L. and C.Z.; Funding acquisition, Z.F. and H.L.; Project administration, Z.F. and H.L.; Writing-original draft, X.L. and C.Z.; Writing—review \& editing, L.Y., Y.Z., Y.W. and H.L. All authors have read and agreed to the published version of the manuscript.

Funding: This work was supported by grants from the National Natural Science Foundation of China (31701927), Central Public-interest Scientific Institution Basal Research Fund (Y2020PT01; Y2018YJ04), Science and Technology Innovation Program of the Chinese Academy of Agricultural Sciences (CAAS-ASTIP-IVFCAAS), and an earmarked fund for the Modern Agro-Industry Technology Research System, China (CARS-23).

Conflicts of Interest: The authors declare no conflict of interest.

\section{References}

1. Vanholme, B.; Grunewald, W.; Bateman, A.; Kohchi, T.; Gheysen, G. The tify family previously known as ZIM. Trends Plant Sci. 2007, 12, 239-244. [CrossRef] [PubMed]

2. Nishii, A.; Takemura, M.; Fujita, H.; Shikata, M.; Yokota, A.; Kohchi, T. Characterization of a novel gene encoding a putative single zinc-finger protein, ZIM, expressed during the reproductive phase in Arabidopsis thaliana. Biosci. Biotechnol. Biochem. 2000, 64, 1402-1409. [CrossRef] [PubMed]

3. Reyes, J.C.; Muro-Pastor, M.I.; Florencio, F.J. The GATA family of transcription factors in Arabidopsis and rice. Plant Physiol. 2004, 134, 1718-1732. [CrossRef] [PubMed]

4. Shikata, M.; Matsuda, Y.; Ando, K.; Nishii, A.; Takemura, M.; Yokota, A.; Kohchi, T. Characterization of Arabidopsis ZIM, a member of a novel plant-specific GATA factor gene family. J. Exp. Bot. 2004, 55, 631-639. [CrossRef]

5. Bateman, A. The Pfam protein families database. Nucleic Acids Res. 2002, 30, 276-280. [CrossRef]

6. Mulder, N.J.; Apweiler, R.; Attwood, T.K.; Bairoch, A.; Bateman, A.; Binns, D.; Bradley, P.; Bork, P.; Bucher, P.; Cerutti, L.; et al. InterPro, progress and status in 2005. Nucleic Acids Res. 2005, 33, D201-D205. [CrossRef]

7. Chung, H.S.; Howe, G.A. A critical role for the TIFY motif in repression of jasmonate signaling by a stabilized splice variant of the JASMONATE ZIM-domain protein JAZ10 in Arabidopsis. Plant Cell 2009, 21, 131-145. [CrossRef] 
8. Bai, Y.; Meng, Y.; Huang, D.; Qi, Y.; Chen, M. Origin and evolutionary analysis of the plant-specific TIFY transcription factor family. Genomics 2011, 98, 128-136. [CrossRef]

9. Ebel, C.; BenFeki, A.; Hanin, M.; Solano, R.; Chini, A. Characterization of wheat (Triticum aestivum) TIFY family and role of Triticum durum TdTIFY11a in salt stress tolerance. PLoS ONE 2018, 13, e0200566. [CrossRef]

10. Sirhindi, G.; Sharma, P.; Arya, P.; Goel, P.; Kumar, G.; Acharya, V.; Singh, A.K. Genome-wide characterization and expression profiling of TIFY gene family in pigeonpea (Cajanus cajan (L.) Millsp.) under copper stress. J. Plant Biochem. Biotechnol. 2016, 25, 301-310. [CrossRef]

11. Wang, Y.; Pan, F.; Chen, D.; Chu, W.; Liu, H.; Xiang, Y. Genome-wide identification and analysis of the Populus trichocarpa TIFY gene family. Plant Physiol. Biochem. 2017, 115, 360-371. [CrossRef] [PubMed]

12. Li, X.Q.; Yin, X.J.; Wang, H.; Li, J.; Guo, C.L.; Gao, H.; Zheng, Y.; Fan, C.H.; Wang, X.P. Genome-wide identification and analysis of the apple (Malus $\times$ domestica Borkh.) TIFY gene family. Tree Genet. Genomes 2015, 11, 808. [CrossRef]

13. Velez-Bermudez, I.C.; Salazar-Henao, J.E.; Fornalé, S.; López-Vidriero, I.; Franco-Zorrilla, J.M.; Grotewold, E.; Gray, J.; Solano, R.; Schmidt, W.; Pagés, M.; et al. A MYB/ZML complex regulates wound-induced lignin genes in Maize. Plant Cell 2015, 27, 3245-3259. [CrossRef]

14. White, D.W. PEAPOD regulates lamina size and curvature in Arabidopsis. Proc. Natl. Acad. Sci. USA 2006, 103, 13238-13243. [CrossRef] [PubMed]

15. Wager, A.; Browse, J. Social Network: JAZ protein interactions expand our knowledge of jasmonate signaling. Front. Plant Sci. 2012, 3, 41. [CrossRef]

16. Kazan, K.; Manners, J.M. JAZ repressors and the orchestration of phytohormone crosstalk. Trends Plant Sci. 2012, 17, 22-31. [CrossRef]

17. Yan, J.; Zhang, C.; Gu, M.; Bai, Z.; Zhang, W.; Qi, T.; Cheng, Z.; Peng, W.; Luo, H.; Nan, F.; et al. The Arabidopsis CORONATINE INSENSITIVE1 protein is a jasmonate receptor. Plant Cell 2009, 21, 2220-2236. [CrossRef]

18. Katsir, L.; Schilmiller, A.L.; Staswick, P.E.; He, S.Y.; Howe, G.A. COI1 is a critical component of a receptor for jasmonate and the bacterial virulence factor coronatine. Proc. Natl. Acad. Sci. USA 2008, 105, 7100-7105. [CrossRef]

19. Lorenzo, O.; Chico, J.M.; Sanchez-Serrano, J.J.; Solano, R. JASMONATE-INSENSITIVE1 encodes a MYC transcription factor essential to discriminate between different jasmonate-regulated defense responses in Arabidopsis. Plant Cell 2004, 16, 1938-1950. [CrossRef]

20. Thines, B.; Katsir, L.; Melotto, M.; Niu, Y.; Mandaokar, A.; Liu, G.; Nomura, K.; He, S.Y.; Howe, G.A.; Browse, J. JAZ repressor proteins are targets of the $\mathrm{SCF}(\mathrm{COI} 1)$ complex during jasmonate signalling. Nature 2007, 448, 661-665. [CrossRef]

21. Chini, A.; Fonseca, S.; Fernández, G.; Adie, B.; Chico, J.M.; Lorenzo, O.; García-Casado, G.; López-Vidriero, I.; Lozano, F.M.; Ponce, M.R.; et al. The JAZ family of repressors is the missing link in jasmonate signalling. Nature 2007, 448, 666-671. [CrossRef] [PubMed]

22. Melotto, M.; Mecey, C.; Niu, Y.; Chung, H.S.; Katsir, L.; Yao, J.; Zeng, W.; Thines, B.; Staswick, P.; Browse, J.; et al. A critical role of two positively charged amino acids in the Jas motif of Arabidopsis JAZ proteins in mediating coronatine- and jasmonoyl isoleucine-dependent interactions with the COI1 F-box protein. Plant J. 2008, 55, 979-988. [CrossRef] [PubMed]

23. Fernández-Calvo, P.; Chini, A.; Fernández-Barbero, G.; Chico, J.M.; Gimenez-Ibanez, S.; Geerinck, J.; Eeckhout, D.; Schweizer, F.; Godoy, M.; Franco-Zorrilla, J.M.; et al. The Arabidopsis bHLH transcription factors MYC3 and MYC4 are targets of JAZ repressors and act additively with MYC2 in the activation of jasmonate responses. Plant Cell 2011, 23, 701-715. [CrossRef] [PubMed]

24. Song, S.; Qi, T.; Huang, H.; Ren, Q.; Wu, D.; Chang, C.; Peng, W.; Liu, Y.; Peng, J.; Xie, D. The Jasmonate-ZIM domain proteins interact with the R2R3-MYB transcription factors MYB21 and MYB24 to affect Jasmonate-regulated stamen development in Arabidopsis. Plant Cell 2011, 23, 1000-1013. [CrossRef] [PubMed]

25. Qi, T.; Song, S.; Ren, Q.; Wu, D.; Huang, H.; Chen, Y.; Fan, M.; Peng, W.; Ren, C.; Xie, D. The Jasmonate-ZIM-domain proteins interact with the WD-Repeat/bHLH/MYB complexes to regulate jasmonate-mediated anthocyanin accumulation and trichome initiation in Arabidopsis thaliana. Plant Cell 2011, 23, 1795-1814. [CrossRef] [PubMed] 
26. Saha, G.; Park, J.I.; Kayum, M.A.; Nou, I.S. A genome-wide analysis reveals stress and hormone responsive patterns of TIFY family genes in Brassica rapa. Front. Plant Sci. 2016, 7, 936. [CrossRef] [PubMed]

27. Ning, Y.; Wang, Y.; Fang, Z.; Zhuang, M.; Zhang, Y.; Lv, H.; Liu, Y.; Li, Z.; Yang, L. Comparative transcriptome analysis of cabbage (Brassica oleracea var. capitata) infected by Plasmodiophora brassicae reveals drastic defense response at secondary infection stage. Plant Soil 2019, 443, 167-183.

28. Liu, X.; Xing, M.; Kong, C.; Fang, Z.; Yang, L.; Zhang, Y.; Wang, Y.; Ling, J.; Yang, Y.; Lv, H. Genetic diversity, virulence, race profiling, and comparative genomic analysis of the Fusarium oxysporum $\mathrm{f}$. sp. conglutinans strains infecting cabbages in China. Front. Microbiol. 2019, 10, 1373.

29. Iglesias-Bernabé, L.; Madloo, P.; Rodríguez, V.M.; Francisco, M.; Soengas, P. Dissecting quantitative resistance to Xanthomonas campestris pv. campestris in leaves of Brassica oleracea by QTL analysis. Sci. Rep. 2019, 9, 2015.

30. Carlsson, M.; Von Bothmer, R.; Merker, A. Screening and evaluation of resistance to downy mildew (Peronospora parasitica) and clubroot (Plasmodiophora brassicae) in genetic resources of Brassica oleracea. Hereditas 2013, 141, 293-300. [CrossRef]

31. Zhang, L.; Zheng, L.; Hsiang, T.; Lv, R.; Huang, J. An outbreak of head rot of cabbage caused by Rhizoctonia solani AG2-1 in central China. Plant Dis. 2009, 93, 109. [CrossRef] [PubMed]

32. Wei, J.F.; Wei, J.H. First report of bacterial soft rot of konnyaku caused by Dickeya dadantii in China. Plant Dis. 2014, 98, 682. [CrossRef] [PubMed]

33. Xing, M.; Lv, H.; Ma, J.; Xu, D.; Li, H.; Yang, L.; Kang, J.; Wang, X.; Fang, Z. Transcriptome profiling of resistance to Fusarium oxysporum $\mathrm{f}$. sp. conglutinans in cabbage (Brassica oleracea) roots. PLoS ONE 2016, 11, e0148048.

34. Liu, S.; Liu, Y.; Yang, X.; Tong, C.; Edwards, D.; Parkin, I.A.; Zhao, M.; Ma, J.; Yu, J.; Huang, S.; et al. The Brassica oleracea genome reveals the asymmetrical evolution of polyploid genomes. Nat. Commun. 2014, 23, 3930. [CrossRef] [PubMed]

35. Lee, J.; Izzah, N.K.; Choi, B.S.; Joh, H.J.; Lee, S.C.; Perumal, S.; Seo, J.; Ahn, K.; Jo, E.J.; Choi, G.J.; et al. Genotyping-by-sequencing map permits identification of clubroot resistance QTLs and revision of the reference genome assembly in cabbage (Brassica oleracea L.). DNA Res. 2015, 23, 29-41. [CrossRef]

36. Liu, X.; Gao, B.; Han, F.; Fang, Z.; Yang, L.; Zhuang, M.; Lv, H.; Liu, Y.; Li, Z.; Cai, C.; et al. Genetics and fine mapping of a purple leaf gene, $B o P r$, in ornamental kale (Brassica oleracea L. var. acephala). BMC Genom. 2017, 18, 230. [CrossRef] [PubMed]

37. Liu, X.; Yu, H.; Han, F.; Li, Z.; Fang, Z.; Yang, L.; Zhuang, M.; Lv, H.; Liu, Y.; Li, Z.; et al. Differentially expressed genes associated with the cabbage yellow-green-leaf mutant in the ygl-1 mapping interval with recombination suppression. Int. J. Mol. Sci. 2018, 19, 2936. [CrossRef]

38. Li, X.; Kong, C.; Yu, H.; Liu, X.; Fang, Z.; Liu, Y.; Yang, L.; Zhuang, M.; Wang, Y.; Lv, H.; et al. Identification of a major QTL for seed number per silique in cabbage (Brassica oleracea L. var. capitata) using genotyping by sequencing. Euphytica 2019, 215, 133.

39. Chen, C.; Xia, R.; Chen, H.; He, Y. TBtools, a toolkit for biologists integrating various HTS-data handling tools with a user-friendly interface. BioRxiv 2018, 289660. [CrossRef]

40. Finn, R.D.; Clements, J.; Eddy, S.R. HMMER web server: Interactive sequence similarity searching. Nucleic Acids Res. 2011, 39, W29-W37. [CrossRef]

41. Marchler-Bauer, A.; Bo, Y.; Han, L.; He, J.; Lanczycki, C.J.; Lu, S.; Chitsaz, F.; Derbyshire, M.K.; Geer, R.C.; Gonzales, N.R.; et al. CDD/SPARCLE: Functional classification of proteins via subfamily domain architectures. Nucleic Acids Res. 2017, 45, D200-D203. [CrossRef] [PubMed]

42. Hu, B.; Jin, J.P.; Guo, A.Y.; Zhang, H.; Luo, J.C.; Gao, G. GSDS 2.0: An upgraded gene feature visualization server. Bioinformatics 2015, 31, 1296-1297. [CrossRef] [PubMed]

43. Bailey, T.L.; Williams, N.; Misleh, C.; Li, W.W. MEME: Discovering and analyzing DNA and protein sequence motifs. Nucleic Acids Res. 2006, 34, 369-373. [CrossRef] [PubMed]

44. Tamura, K.; Stecher, G.; Peterson, D.; Filipski, A.; Kumar, S. MEGA6: Molecular evolutionary genetics analysis version 6.0. Mol. Biol. Evol. 2013, 30, 2725-2729. [CrossRef]

45. Trapnell, C.; Williams, B.A.; Pertea, G.; Mortazavi, A.; Kwan, G.; van Baren, M.J.; Salzberg, S.L.; Wold, B.J.; Pachter, L. Transcript assembly and quantification by RNA-Seq reveals unannotated transcripts and isoform switching during cell differentiation. Nat. Biotechnol. 2010, 28, 511-515. [CrossRef] 
46. Livak, K.J.; Schmittgen, T.D. Analysis of relative gene expression data using real-time quantitative PCR and the $2^{-\Delta \Delta C t}$ method. Methods 2001, 25, 402-408. [CrossRef]

47. Rieu, I.; Powers, S.J. Real-time quantitative RT-PCR: Design, calculations, and statistics. Plant Cell 2009, 21, 1031-1033. [CrossRef]

48. Voinnet, O.; Lederer, C.; Baulcombe, D.C. A viral movement protein prevents systemic spread of the gene silencing signal in Nicotiana benthamiana. Cell 2000, 103, 157-167. [CrossRef]

49. Lysak, M.A.; Cheung, K.; Kitschke, M.; Bures, P. Ancestral chromosomal blocks are triplicated in Brassiceae species with varying chromosome number and genome size. Plant Physiol. 2007, 145, 402-410. [CrossRef]

50. Thatcher, L.F.; Cevik, V.; Grant, M.; Zhai, B.; Jones, J.D.; Manners, J.M.; Kazan, K. Characterization of a JAZ7 activation-tagged Arabidopsis mutant with increased susceptibility to the fungal pathogen Fusarium oxysporum. J. Exp. Bot. 2016, 67, 2367-2386. [CrossRef]

51. Robert-Seilaniantz, A.; Grant, M.; Jones, J.D. Hormone crosstalk in plant disease and defense: More than just jasmonate-salicylate antagonism. Annu. Rev. Phytopathol. 2011, 49, 317-343. [CrossRef] [PubMed]

52. Huang, H.; Liu, B.; Liu, L.; Song, S. Jasmonate action in plant growth and development. J. Exp. Bot. 2017, 68, 1349-1359. [CrossRef] [PubMed]

53. The Arabidopsis Genome Initiative. Analysis of the genome sequence of the flowering plant Arabidopsis thaliana. Nature 2000, 408, 796-815. [CrossRef] [PubMed]

54. Murat, F.; Louis, A.; Maumus, F.; Armero, A.; Cooke, R.; Quesneville, H.; Crollius, H.R.; Salse, J. Erratum to: Understanding Brassicaceae evolution through ancestral genome reconstruction. Genome Biol. 2016, 17, 64. [CrossRef]

55. Warwick, S.I.; Mummenhoff, F.; Sauder, C.A.; Koch, M.A.; Al-Shehbaz, I.A. Closing the gaps: Phylogenetic relationships in the Brassicaceae based on DNA sequence data of nuclear ribosomal ITS region. Plant Systemat. Evol. 2010, 285, 209-232. [CrossRef]

56. Koenig, D.; Weigel, D. Beyond the thale: Comparative genomics and genetics of Arabidopsis relatives. Nat. Rev. Genet. 2015, 16, 285-298. [CrossRef]

57. Zhang, L.; Cai, X.; Wu, J.; Liu, M.; Grob, S.; Cheng, F.; Liang, J.; Cai, C.; Liu, Z.; Liu, B.; et al. Improved Brassica rapa reference genome by single-molecule sequencing and chromosome conformation capture technologies. Hortic. Res. 2018, 5, 50. [CrossRef]

58. Lysak, M.A.; Koch, M.A.; Beaulieu, J.M.; Meister, A.; Leitch, I.J. The dynamic ups and downs of genome size evolution in Brassicaceae. Mol. Biol. Evol. 2009, 26, 85-98. [CrossRef]

59. Demianski, A.J.; Chung, K.M.; Kunkel, B.N. Analysis of Arabidopsis JAZ gene expression during Pseudomonas syringae pathogenesis. Mol. Plant Pathol. 2012, 13, 46-57. [CrossRef]

60. Ye, H.; Du, H.; Tang, N.; Li, X.; Xiong, L. Identification and expression profiling analysis of TIFY family genes involved in stress and phytohormone responses in rice. Plant Mol. Biol. 2009, 71, 291-305. [CrossRef]

61. Freeling, M. Bias in plant gene content following different sorts of duplication: Tandem, whole-genome, segmental, or by transposition. Annu. Rev. Plant Biol. 2009, 60, 433-453. [CrossRef] [PubMed]

62. Wang, N.; Xiang, Y.; Fang, L.; Wang, Y.; Xin, H.; Li, S. Patterns of gene duplication and their contribution to expansion of gene families in grapevine. Plant Mol. Biol. Rep. 2013, 31, 852-861. [CrossRef]

63. Jiao, Y.; Leebens-Mack, J.; Ayyampalayam, S.; Bowers, J.E.; McKain, M.R.; McNeal, J.; Rolf, M.; Ruzicka, D.R.; Wafula, E.; Wickett, N.J.; et al. Depamphilis, A genome triplication associated with early diversification of the core eudicots. Genome Biol. 2012, 13, R3. [CrossRef] [PubMed]

64. Xia, W.; Yu, H.; Cao, P.; Luo, J.; Wang, N. Identification of TIFY family genes and analysis of their expression profiles in response to phytohormone treatments and Melampsora larici-populina infection in poplar. Front. Plant Sci. 2017, 8, 493. [CrossRef] [PubMed]

65. Pozo, M.J.; Van Loon, L.C.; Pieterse, C.M.J. Jasmonates-Signals in plant-microbe interactions. J. Plant Growth Regul. 2004, 23, 211-222.

66. Balbi, V.; Devoto, A. Jasmonate signalling network in Arabidopsis thaliana: Crucial regulatory nodes and new physiological scenarios. New Phytol. 2008, 177, 301-318. [CrossRef]

67. Chini, A.; Fonseca, S.; Chico, J.M.; Fernández-Calvo, P.; Solano, R. The ZIM domain mediates homo- and heteromeric interactions between Arabidopsis JAZ proteins. Plant J. 2009, 59, 77-87. [CrossRef]

68. Thireault, C.; Shyu, C.; Yoshida, Y.; St Aubin, B.; Campos, M.L.; Howe, G.A. Repression of jasmonate signaling by a non-TIFY JAZ protein in Arabidopsis. Plant J. 2015, 82, 669-679. [CrossRef] 
69. Staswick, P.E.; Su, W.; Howell, S.H. Methyl jasmonate inhibition of root growth and induction of a leaf protein are decreased in an Arabidopsis thaliana mutant. Proc. Natl. Acad. Sci. USA 1992, 89, 6837-6840. [CrossRef]

70. Pieterse, C.M.; Dicke, M. Plant interactions with microbes and insects: From molecular mechanisms to ecology. Trends Plant Sci. 2007, 12, 564-569. [CrossRef]

71. Mur, L.A.; Laarhoven, L.J.; Harren, F.J.; Hall, M.A.; Smith, A.R. Nitric oxide interacts with salicylate to regulate biphasic ethylene production during the hypersensitive response. Plant Physiol. 2008, 148, 1537-1546. [CrossRef] [PubMed]

72. Lin, Z.F.; Zhong, S.L.; Grierson, D. Recent advances in ethylene research. J. Exp. Bot. 2009, 60, 3311-3336. [CrossRef] [PubMed]

73. Gfeller, A.; Liechti, R.; Farmer, E.E. Arabidopsis jasmonate signaling pathway. Sci. Signal. 2006, 3, cm4. [CrossRef] [PubMed]

74. Mur, L.A.; Prats, E.; Pierre, S.; Hall, M.A.; Hebelstrup, K.H. Integrating nitric oxide into salicylic acid and jasmonic acid/ethylene plant defense pathways. Front. Plant Sci. 2013, 4, 215. [CrossRef]

75. Zhu, Z.; An, F.; Feng, Y.; Li, P.; Xue, L.; Abu-El-Haija, A.; Jiang, Z.; Kim, J.M.; To, T.K.; Li, W.; et al. De-repression of ethylene-stabilized transcription factors (EIN3/EIL1) mediates jasmonate and ethylene signaling synergy in Arabidopsis. Proc. Natl. Acad. Sci. USA 2011, 108, 12539-12544. [CrossRef]

76. Chen, H.; Xue, L.; Chintamanani, S.; Germain, H.; Lin, H.; Cui, H.; Cai, R.; Zuo, J.; Tang, X.; Li, X.; et al. ETHYLENE INSENSITIVE3 and ETHYLENE INSENSITIVE3-LIKE1 repress SALICYLIC ACID INDUCTION DEFICIENT2 expression to negatively regulate plant innate immunity in Arabidopsis. Plant Cell 2009, 21, 2527-2540. [CrossRef]

77. Cheng, H.; Song, S.; Xiao, L.; Soo, H.M.; Cheng, Z.; Xie, D.; Peng, J. Gibberellin acts through jasmonate to control the expression of MYB21, MYB24, and MYB57 to promote stamen filament growth in Arabidopsis. PLoS Genet. 2009, 5, e1000440. [CrossRef]

78. Pauwels, L.; Inzé, D.; Goossens, A. Jasmonate-inducible gene: What does it mean? Trends Plant Sci. 2009, 14, 87-91. [CrossRef]

79. Hou, X.; Lee, L.Y.; Xia, K.; Yan, Y.; Yu, H. DELLAs modulate jasmonate signaling via competitive binding to JAZs. Dev. Cell 2011, 19, 884-894. [CrossRef]

80. Navarro, L.; Bari, R.; Achard, P.; Lisón, P.; Nemri, A.; Harberd, N.P.; Jones, J.D. DELLAs control plant immune responses by modulating the balance of jasmonic acid and salicylic acid signaling. Curr. Biol. 2008, 18, 650-655. [CrossRef]

81. Lemarie, S.; Robert-Seilaniantz, A.; Lariagon, C.; Lemoine, J.; Marnet, N.; Jubault, M.; Manzanares-Dauleux, M.J.; Gravot, A. Both the jasmonic acid and the salicylic acid pathways contribute to resistance to the biotrophic clubroot agent Plasmodiophora brassicae in Arabidopsis. Plant Cell Physiol. 2015, 56, 2158-2168. [PubMed]

82. Liu, T.; Zhang, X.; Yang, H.; Agerbirk, N.; Qiu, Y.; Wang, H.; Shen, D.; Song, J.; Li, X. Aromatic glucosinolate biosynthesis pathway in Barbarea vulgaris and its response to Plutella xylostella infestation. Front. Plant Sci. 2016, 7, 83. [CrossRef] [PubMed]

83. Xu, L.; Yang, H.; Ren, L.; Chen, W.; Liu, L.; Liu, F.; Zeng, L.; Yan, R.; Chen, K.; Fang, X. Jasmonic acid-mediated aliphatic glucosinolate metabolism is involved in clubroot disease development in Brassica napus L. Front. Plant Sci. 2018, 9, 750. [CrossRef] [PubMed]

(C) 2020 by the authors. Licensee MDPI, Basel, Switzerland. This article is an open access article distributed under the terms and conditions of the Creative Commons Attribution (CC BY) license (http://creativecommons.org/licenses/by/4.0/). 